\title{
Neurotoxic Lesions of Basolateral, But Not Central, Amygdala Interfere with Pavlovian Second-Order Conditioning and Reinforcer Devaluation Effects
}

\author{
Tammy Hatfield, ${ }^{1}$ Jung-Soo Han, ${ }^{1}$ Michael Conley, ${ }^{2}$ Michela Gallagher, ${ }^{1}$ and Peter Holland ${ }^{2}$ \\ ${ }^{1}$ Department of Psychology, University of North Carolina at Chapel Hill, Chapel Hill, North Carolina 27599, and \\ 2Department of Psychology-Experimental, Duke University, Durham, North Carolina 27708
}

\begin{abstract}
Considerable evidence suggests that various discrete nuclei within the amygdala complex are critically involved in the assignment of emotional significance or value to events through associative learning. Much of this evidence comes from aversive conditioning procedures. For example, lesions of either basolateral amygdala (ABL) or the central nucleus $(\mathrm{CN})$ interfere with the acquisition or expression of conditioned fear. The present study examined the effects of selective neurotoxic lesions of either $\mathrm{ABL}$ or $\mathrm{CN}$ on the acquisition of positive incentive value by a conditioned stimulus (CS) with two appetitive Pavlovian conditioning procedures. In second-order conditioning experiments, rats first received light-food pairings intended to endow the light with reinforcing power. The acquired reinforcing power of the light was then measured by examining its ability to serve as a reinforcer for second-order conditioning of a tone when tone-light pairings were given in the absence of food. Acquisition of second-order conditioning
\end{abstract}

was impaired in rats with $A B L$ lesions but not in rats with $C N$ lesions. In reinforcer devaluation procedures, conditioned responding of rats with $\mathrm{ABL}$ lesions was insensitive to postconditioning changes in the value of the reinforcer, whereas rats with $\mathrm{CN}$ lesions, like normal rats, were able to spontaneously adjust their CRs to the current value of the reinforcer. The results of both test procedures indicate that $A B L$, but not $C N$, is part of a system involved in CSs' acquisition of positive incentive value. Together with evidence that identifies a role for $\mathrm{CN}$ in certain changes in attentional processing of CSs in conditioning, these results suggest that separate amygdala subsystems contribute to a variety of processes inherent in associative learning.

Key words: basolateral amygdala; amygdala central nucleus; second-order conditioning; reinforcer devaluation; classical conditioning; rats
Much evidence indicates that neural processing in the amygdala complex is important for assigning emotional value or significance to events through associative learning. For example, cues that signal an aversive event elicit freezing and potentiate startle reactivity and produce characteristic autonomic responses in rodents. Discrete lesions or inactivation of basolateral amygdala $(\mathrm{ABL})$ or the central nucleus $(\mathrm{CN})$ produce deficits in the acquisition and/or expression of a range of conditioned fear behaviors (for review, see Davis, 1992; LeDoux, 1992). Similarly, unlike normal rats, rats with $\mathrm{ABL}$ lesions typically fail to acquire preferences for environmental locations paired with positively reinforcing events, such as food or certain drug states (Everitt and Robbins, 1992; Everitt et al., 1991; McDonald and White, 1993).

Recent research indicates that the amygdala also serves an attentional function in Pavlovian appetitive conditioning. Rats with selective neurotoxic lesions of the $\mathrm{CN}$ show a pronounced deficit in the acquisition of conditioned orienting behavior to visual and auditory conditioned stimuli (CSs) paired with food (Gallagher et al., 1990). Furthermore, a variety of experimental manipulations that produce more general enhancement of attentional processing of CSs in nor-

Received March 6, 1996; revised June 10, 1996; accepted June 14, 1996.

This work was supported by grants from the National Institute of Mental Health (K05 MH01149 and R01 MH53667) and the Human Frontier Science Research Program to M.G. and P.C.H. We thank John Chen, Veronica Davi, Christina Ewing, Ayana Ferguson, and P. Nicole Fielder for technical assistance.

Correspondence should be addressed to Peter Holland, Department of Psychology-Experimental, P.O. Box 90086, Duke University, Durham, NC 277080086.

Copyright (C) 1996 Society for Neuroscience $0270-6474 / 96 / 165256-10 \$ 05.00 / 0$ mal rats fail to affect the behavior of rats with $\mathrm{CN}$ lesions (Holland and Gallagher, 1993a,b). At the same time, evidence from those studies suggests that these attentional functions are regulated by the $\mathrm{CN}$ independently of other processes involved in appetitive conditioning. First, rats with $\mathrm{CN}$ lesions were unimpaired in their acquisition of conditioned responses (CRs) directed to the food cup, similar to the behaviors controlled by the food unconditioned stimulus (US) itself (Gallagher et al., 1990). Second, rats with $\mathrm{CN}$ damage and normal rats were equally sensitive to variations in US magnitude when shifts in attention were not involved (Holland and Gallagher, 1993b). Thus, rats with $\mathrm{CN}$ damage apparently remained sensitive to the incentive value of the US.

These data led Gallagher and Holland (1994) to propose that separate amygdala subsystems mediate attentional processes and changes in the incentive value of cues in Pavlovian appetitive conditioning. The experiments reported here examined that dissociation more explicitly by examining the effects of discrete lesions of ABL (Experiment 1) and CN (Experiment 2) on conditioned orienting and on two measures of the transfer of incentive value from the US to the CS, Pavlovian second-order conditioning, and US devaluation. We anticipated that CN lesions, but not $\mathrm{ABL}$ lesions, would interfere with the acquisition of conditioned orienting, and that $\mathrm{ABL}$ lesions, but not $\mathrm{CN}$ lesions, would interfere with CSs' acquisition of incentive value.

\section{EXPERIMENT 1A}

The most common assessment of whether an event has acquired incentive value in conditioning is the measurement of its ability to 
serve as a reinforcer in new learning (Mackintosh, 1983). In Experiment 1A, we evaluated a CS's acquired reinforcement value with a Pavlovian second-order conditioning procedure (Holland and Rescorla, 1975). In this procedure, rats first received light-food pairings intended to endow the light with reinforcing power, and then in a second phase, they received second-order tone-light pairings, in the absence of food. Acquisition of Pavlovian second-order conditioning to the tone CS provided a measure of the reinforcing power of the light. If $\mathrm{ABL}$ lesions interfered with the light's acquisition of conditioned reinforcement value, then lesioned rats would fail to acquire second-order conditioning to the tone, even if they exhibited substantial CRs to the light in the first conditioning phase.

The design of the study included procedures to demonstrate that responding to the tone was dependent on associative processes. Control subjects that received tone-light pairings in the second phase but no light-food pairings in the first phase were included to show that the ability of the light to reinforce secondorder conditioning to the tone depended on previous light-food pairings and, hence, reflected learned reinforcing value, as distinct from some primary, unlearned value of the light. Similarly, other control rats that received light-food pairings in the first phase, but explicitly unpaired tone and light presentations pairings in the second phase, showed that responding to the tone was the result of associative learning in the second phase, rather than some nonassociative process such as sensitization.

\section{Materials and methods}

Subjects. Eighty-five experimentally naive male Long-Evans rats (300$350 \mathrm{gm}$ ) that were obtained from Charles River Breeding Laboratories (Raleigh, NC) served as subjects. Rats were individually housed in plastic cages with access to food and water ad libitum until 1 week after surgery. Then the rats were transferred to individual stainless steel cages and, after access to food ad libitum for an additional week, were gradually reduced to $85 \%$ of their ad libitum weights by limiting their access to food; water was always available. The rats were weighed and fed daily to maintain their $85 \%$ weights for the remainder of the experiment. The vivarium in which the rats were housed was maintained at $21^{\circ} \mathrm{C}$, with the lights on from 6:00 A.M. to 8:00 P.M. daily. All experimental sessions were carried out during the light portion of the cycle, between 7:00 A.M. and 2:00 P.M.

Apparatus. The apparatus consisted of eight individual chambers $(22.9 \times 20.3 \times 20.3 \mathrm{~cm})$ with aluminum front and back walls, clear acrylic sides and top, and a grid floor $(0.48 \mathrm{~cm}$ stainless steel rods spaced $1.9 \mathrm{~cm}$ apart). A dimly illuminated food cup was recessed in the center of one end wall; a jeweled lamp (not used in this study) was located $5 \mathrm{~cm}$ above that recess. Each experimental chamber was enclosed in a sound-resistant shell with an acrylic window for viewing the rats. A $6 \mathrm{~W}$ normally-off house light, the illumination of which served as the visual ("light") CS, was mounted on the inside wall of the shell, $25 \mathrm{~cm}$ above and behind the experimental chamber, even with the end wall that contained the food cup. A speaker, used to present the auditory CS, was mounted next to the house light. Ventilation fans provided masking noise $(70 \mathrm{~dB})$, and a $6 \mathrm{~W}$, $110 \mathrm{~V}$ lamp (operated at $75 \mathrm{~V}$ ) behind a red lens opposite the house light provided continuous dim background illumination. Two low-light television cameras were mounted $2.1 \mathrm{~m}$ from the experimental chambers so each could include four chambers in its field of view. VCRs were programmed to record behaviors that occurred during the $10 \mathrm{sec}$ intervals before, during, and after CS presentations.

Surgery. Rats were anesthetized with Nembutal (sodium pentobarbital; $50 \mathrm{mg} / \mathrm{kg}$, i.p.) for stereotaxic surgery. Bilateral lesions of ABL were made using the following stereotaxic coordinates: $2.8 \mathrm{~mm}$ posterior from bregma and $5.0 \mathrm{~mm}$ from the midline, with two injection sites ventral from the skull surface at $8.4 \mathrm{~mm}(0.2 \mu \mathrm{l})$ and $8.1 \mathrm{~mm}(0.1 \mu \mathrm{l})$ (Paxinos and Watson, 1986). The ABL lesions were made using NMDA $(20 \mathrm{mg} / \mathrm{ml})$ in a Krebs-Ringer phosphate solution, $\mathrm{pH}$ 7.4, which was injected using a Hamilton $1.0 \mu \mathrm{l}$ syringe at a rate of $0.2 \mu \mathrm{l} / 30 \mathrm{sec}$. Control animals were given vehicle injections. Injector needles were left in place at each injection site for $3 \mathrm{~min}$. Fifty-eight rats received bilateral lesions of the $\mathrm{ABL}$, and 27 rats served as controls. All subjects were allowed to recover
Table 1. Pavlovian second-order conditioning procedure used in Experiments 1A and 2A

\begin{tabular}{|c|c|c|c|c|}
\hline \multirow[b]{2}{*}{ Group } & \multirow{2}{*}{$\begin{array}{l}\text { Phase } 1 \\
\begin{array}{l}\text { First-order } \\
\text { conditioning }\end{array}\end{array}$} & \multicolumn{3}{|c|}{ Phase 2} \\
\hline & & $\begin{array}{l}\text { Pretest } \\
\text { tone }\end{array}$ & $\begin{array}{l}\text { Second-order } \\
\text { conditioning }\end{array}$ & $\begin{array}{l}\text { Reminder } \\
\text { trials }\end{array}$ \\
\hline PP & Light $\rightarrow$ food & Tone & Tone $\rightarrow$ light & Light $\rightarrow$ food \\
\hline PU & Light $\rightarrow$ food & Tone & Tone, light & Light $\rightarrow$ food \\
\hline UP & Light, food & Tone & Tone $\rightarrow$ light & Light, food \\
\hline
\end{tabular}

The tone pretest occurred during the first half of the first session of Phase 2 . Second-order conditioning and first-order reminder trials were randomly intermixed throughout the remainder of Phase 2 . $\rightarrow$, Paired presentation; , indicates explicitly unpaired presentation.

postoperatively for 2 weeks with access to food and water ad libitum before behavioral testing.

Behavioral testing. Table 1 gives a summary of the behavioral testing procedures. Before training, each rat was taught to eat from the food cup. In one $64 \mathrm{~min}$ session, there were 16 presentations of the food reinforcer, delivered on a variable-time 4 min schedule. The reinforcer used throughout this experiment was the delivery of two $45 \mathrm{mg}$ food pellets (P.J. Noyes Co.), separated by $0.5 \mathrm{sec}$.

Phase 1 was designed to establish first-order conditioning to a visual CS in two groups of rats, but to leave that stimulus unconditioned in a third group. In each of the eight, 64 min sessions, the rats in Groups $P \mathrm{P}$ and $P \mathrm{U}$ received $P$ aired presentations of a $10 \mathrm{sec}$ intermittent $(3 \mathrm{~Hz})$ illumination of the house light; the food reinforcer was delivered immediately after the termination of the light. In each of those sessions, the rats in Group $U \mathrm{P}$ received eight light and eight food presentations, but those events were explicitly Unpaired.

Phase 2 was designed to establish second-order conditioning to a tone CS in Group PP, but to leave that tone unconditioned in Groups PU and UP. Rats in Groups PP and UP received tone-light $P$ airings, in which a $10 \mathrm{sec}, 1500 \mathrm{~Hz}$ tone was followed immediately by the light CS. The rats in Group $\mathrm{P} U$ received tone and light presentations Unpaired. Thus, only Group PP received pairings of the second-order tone CS with a previously conditioned light first-order CS. The rats in Group UP received pairings of the tone with a light that was not previously conditioned, controlling for any natural reinforcing power the light might possess, and the rats in Group PU did not receive pairings of the tone with the light, controlling for any unconditioned effects of tone, light, and food presentations.

In the first half of the first session of second-order conditioning, all rats received three $10 \mathrm{sec}$ presentations of the tone alone as a pretest of the unconditioned effects of that stimulus. In addition, each rat received one "reminder" presentation of the light and food identical to those of the preceding phase. Thus, rats in Groups PP and PU received a light-food pairing, and the rats in Group UP received one light-alone and one food-alone presentation. In the second half of this session, the rats in Groups PP and UP received three tone-light pairings, and one reminder presentation of light and food, as before, and the rats in Group PU received three tone-alone presentations, three light-alone presentations, and one light-food reminder pairing. Each half of the remaining two sessions of second-order conditioning was identical to this half-session.

Behavioral observations. All observations were made from videotapes and paced by auditory signals recorded on the tapes. For each rat, observations were made at $1.25 \mathrm{sec}$ intervals during the $5 \mathrm{sec}$ period immediately before CS presentations and during CS presentations. At each observation, one and only one behavior was recorded.

The primary measure of learning used in this experiment was food-cup behavior, which occurred in response to both the first-order visual CS and the second-order auditory CS. Food-cup behavior includes standing motionless in front of the recessed food cup, with the head or nose within the recessed area, and head-jerk behavior (short, rapid horizontal and/or vertical movements of the head). In addition, three other behaviors were reported. Rear behavior (standing on hind legs with front feet off the floor, and not grooming) is the orienting response (OR) specific to visual CSs. Startle behavior (a jump that occurs in response to CS onset) is the OR specific to auditory CSs. Note that it is not known how this behavioral category (described by Holland, 1977) relates to the frequently studied acoustic startle reflex (Davis, 1992), which is often defined much more precisely in terms of latency and waveform. Walk behavior (walking, running, or circling) occurs as a component of second-order CRs during 
auditory cues [see Holland (1977), Exp. 2] and was observed only in Phase 2.

Previous data (Holland, 1977) showed that rear behavior tends to occur mostly during the early portions of $10 \mathrm{sec}$ visual cues and that food-cup behavior occurs primarily in the later portions. Consequently, we report the occurrence of rear behavior during the first $5 \mathrm{sec}$ and food-cup behavior during the last $5 \mathrm{sec}$ interval of $10 \mathrm{sec}$ visual CS presentations. In contrast, both food-cup and walk behaviors tend to be relatively evenly distributed during $10 \mathrm{sec}$ auditory CSs, and so we report their frequency over the entire $10 \mathrm{sec}$ CS interval.

The index of behavioral frequency used was percentage total behavior, obtained by dividing the frequency of the target behavior (e.g., food cup) in any observation interval by the total number of observations made in that interval. Note that because the rate of observations was constant within each observation interval throughout the experiments, this measure corresponds to an absolute frequency measure: it is not affected by the overall activity level of a subject. Because startle behavior to the tone CS could only occur once on a trial, the measure of startle responding was the percentage of trials on which that behavior occurred. Six observers (J.C., V.D., C.E., A.F., P.N.F., and P.C.H.) scored the behavioral data reported in these experiments. All Phase 2 data were scored by P.C.H.; Phase 1 data were scored by C.E. and P.C.H. in Experiment 1, and by J.C., V.D., A.F., P.N.F., and P.C.H. in Experiment 2. To assess objectivity, the data from several randomly selected sessions in each phase of the experiment were scored by both P.C.H. and one of the other observers. The two observers agreed on $89 \%$ of over 8000 joint observations. No observer was aware of the lesion conditions of the rats when the data were scored.

For the statistical analyses of all measures, we used two-tailed distribution-free statistics. We adopted $p<0.05$ as the level of significance.

\section{Results and discussion Histology}

A representative $\mathrm{ABL}$ lesion is shown in Figure 1. Histological analysis of the ABL revealed that 19 of the 58 lesioned rats had neuronal loss that was confined bilaterally to the ABL (including basal and lateral nuclei). The remaining animals in the lesion group had either predominantly unilateral damage or insufficient damage to the ABL. Only data from the 19 rats with bilateral damage confined to the ABL were used in the analysis. The acceptable lesions ranged in size from 40 to $100 \%$ of the total $\mathrm{ABL}$ area. The average lesion encompassed $\sim 74 \%$. In all cases, the lesion sites were marked with gliosis, and intact neurons were clearly visible at the borders of the lesions. There was no detectable loss of neurons in the $\mathrm{CN}$ for this group. In some cases, there was damage outside the ABL that included substantial amounts of damage to the piriform cortex; however, there were no behavioral differences between groups with ABL alone or ABL plus piriform damage. No animals were excluded from the control group: injector tracts were visible in all cases. Thus, data for 19 ABLlesioned and 27 ABL-control rats were analyzed.

\section{Behavior}

In phase 1, first-order conditioning was acquired rapidly to the light CS when it was paired with food (Groups PP and PU) in both unlesioned and ABL-lesioned rats. Figure 2 shows performance of the first-order CRs and conditioned ORs during the light CS on light-food or light-alone reminder trials over the course of Phase 2. The left side of Figure 2 shows first-order food-cup CRs. Both ABL-lesioned (Mann-Whitney $U_{(14,5)}=$ 1.5) and unlesioned $\left(U_{(19,8)}=0\right)$ rats in Groups PP and PU (combined; open bars) showed reliably more responding than those in Group UP (solid bars), in which the light and food were explicitly unpaired. Food-cup responding of lesioned and intact rats did not differ in either the paired $\left(U_{(14,19)}=104.5\right)$ or the unpaired $\left(U_{(5,8)}=19\right)$ rats.

The right side of Figure 2 shows first-order conditioned ORs
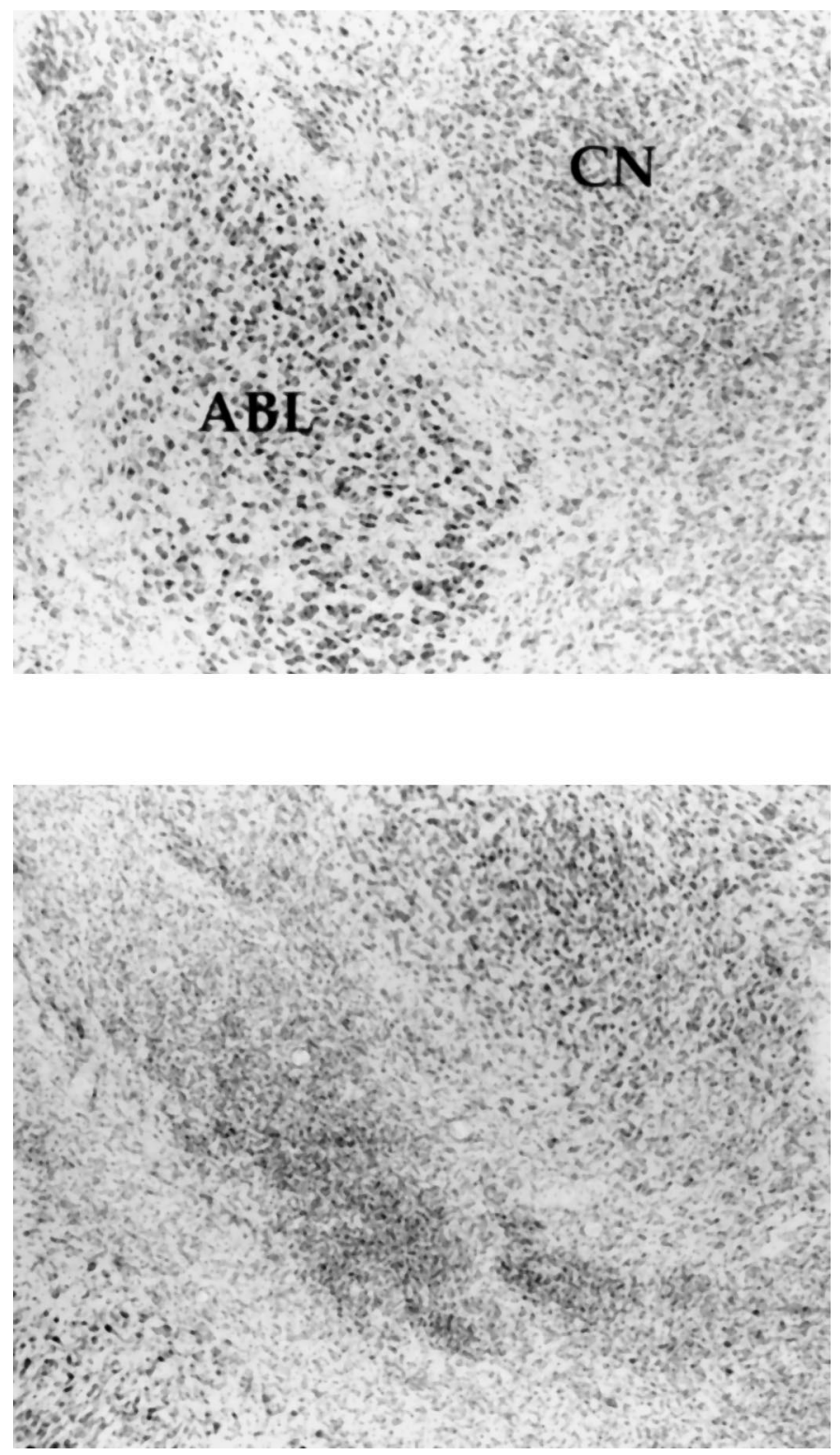

Figure 1. Photomicrographs showing the region of basolateral amygdala $(A B L)$ and amygdala central nucleus $(C N)$ in a vehicle-injected control brain (top panel) and in an ABL NMDA-lesioned brain (bottom panel). Note neuron loss and gliosis at the $A B L$ lesion site, and sparing of neurons in $C N$.

(rear behavior). Both ABL-lesioned $\left(U_{(14,5)}=14\right)$ and unlesioned $\left(U_{(19,8)}=18\right)$ rats in Groups PP and PU (combined) showed reliably more rear behavior than those in Group UP. As with food-cup responding, rear responding of ABL-lesioned and intact rats did not differ in either the paired $\left(U_{(14,19)}=129.5\right)$ or the unpaired $\left(U_{(5,8)}=16\right)$ rats. However, the claim that ABL lesions had no effect on conditioned orienting is weakened by the low levels of rear behavior displayed by both lesioned and unlesioned rats in this experiment, compared to that observed in other studies (Gallagher et al., 1990) (this work, Experiment 2).

Figure 3 shows the primary behavioral data of Experiment 2A, the acquisition of second-order conditioning to the tone CS in Phase 2. The left side of Figure 3 shows the acquisition of second-order CRs (food-cup and walk behaviors, combined), and the right side shows the acquisition of second-order ORs (startle 


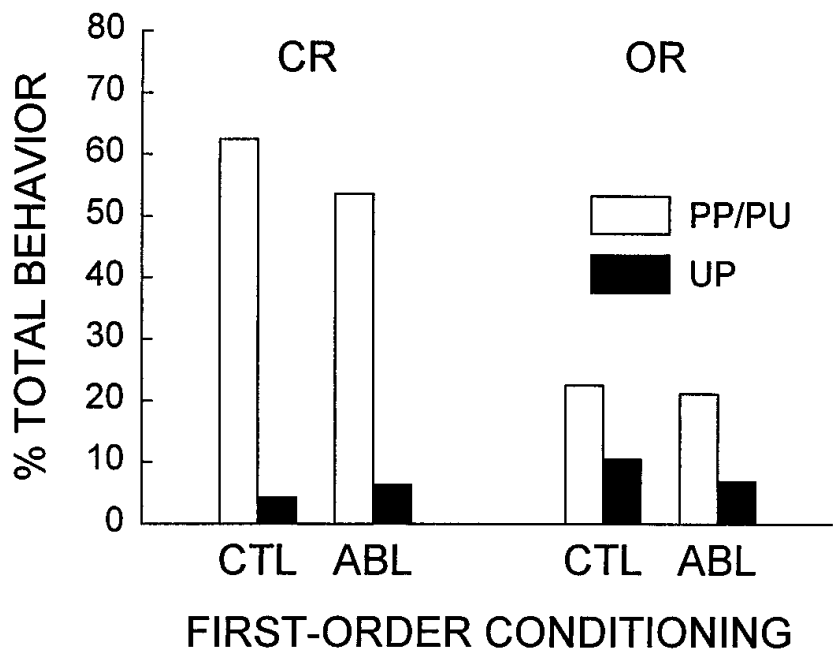

Figure 2. First-order conditioned responses displayed by rats with basolateral amygdala lesions $(A B L)$ and unlesioned control rats $(C T L)$ during the light reminder trials in Phase 2 of Experiment 1A. Combined performance of the rats that received light-food pairings (Groups $P P$ and $P U$ ) is indicated by the open bars and performance of the rats that received unpaired presentations of light and food (Group UP) is indicated by the solid bars.

behavior). The ABL lesions disrupted second-order conditioning: in Group PP, only the unlesioned rats showed evidence for acquisition. Both second-order CRs $\left(U_{(10,9)}=21.5\right)$ and second-order ORs $\left(U_{(10,9)}=21\right)$ were significantly more frequent in the unlesioned rats than in the ABL-lesioned rats in that group. Furthermore, although among unlesioned rats, both second-order CRs $\left(U_{(9,18)}=39\right)$ and second-order ORs $\left(U_{(9,18)}=41\right)$ were reliably more frequent in Group PP than in the two control groups (combined), responding of ABL-lesioned rats in Group PP was not reliably greater than that of the ABL-lesioned rats in the control groups $\left(U_{(10,9)} \geq 31.5\right)$.

Thus, ABL lesions prevented the acquisition of second-order conditioning in this experiment, suggesting that light-food pairings in the first phase did not endow that light with reinforcement value in the lesioned rats. At the same time, the lack of a reliable effect of the ABL lesions on the acquisition of first-order CRs shows that the inability of the lesioned rats to learn second-order conditioning was not the consequence of a general impairment in learning ability. (In that respect, it is worth noting that the moderate level of first-order responding observed in Phase 2 makes it unlikely that lesion-induced differences in first-order conditioning were masked by ceiling effects.) Instead, the ABL lesion deficit was limited to the light inability of CSs to serve as a reinforcer for second-order conditioning of the tone. Thus, these results also indicate that CSs' acquisition of reinforcement value and of the ability to evoke CRs are anatomically distinguishable; only the former is mediated by ABL.

The impairment in second-order conditioning produced by ABL damage is consistent with the deficit in secondary reinforcement learning observed by Everitt and Robbins (1992) in ABLlesioned rats. Our use of a Pavlovian second-order conditioning procedure, however, avoids a potential confound inherent in secondary reinforcement procedures like those used by Everitt and Robbins (1992). In those procedures, the acquisition of value as a consequence of Pavlovian conditioning in the first phase is assessed by examining the ability of the CS to serve as a reinforcer for operant conditioning of an arbitrary response in the second phase. Consequently, lesion-produced disruption of secondary reinforcement learning might reflect selective effects of the lesion on operant learning processes, rather than on the CS's acquisition of emotional value in the initial the Pavlovian learning phase. Indeed, Killcross et al. (1995) have recently shown differential effects of amygdala lesions on operant and Pavlovian learning in related paradigms. Although it might be similarly argued that ABL lesions selectively interfere with Pavlovian second-order associative processes, but leave CS value intact, we interpret the results of Experiment 1 as implicating ABL involvement in the transfer of incentive value from the US to the CS in appetitive conditioning. The results of Experiment 1B support this interpretation.

\section{EXPERIMENT 1B}

Modern views of Pavlovian conditioning (for a brief review, see Holland, 1993) often presume that the production of CRs is mediated by CSs' gaining access to some internal representation of the US. These views are supported by considerable data that indicate that responding to CSs is often sensitive to post-training alterations in the value of the US. For example, Holland and Straub (1979) first presented rats with pairings of a tone CS with a food pellet US. Then, in the absence of the tone, food pellets were paired with the administration of a toxin, lithium chloride $(\mathrm{LiCl})$. As a result, the rats formed an aversion to the food pellets and would not consume them in brief consumption tests. A subsequent test of conditioned responding to the tone CS (in the absence of food delivery) showed an analogous, spontaneous drop in CRs as well. These results can be interpreted as implying that the CS acquires not just the value of the US at the time of conditioning, but the ability to gain access to the current value of the US at the time of performance.

Experiment $1 \mathrm{~B}$ considered whether ABL lesions interfere with the ability of CSs to gain access to the current value of the US. All rats from Experiment 1 that had received light-food pairings (Groups PP and PU) were redistributed into two new groups. One group received two pairings of the food pellets (which had served as the US) with the injection of $\mathrm{LiCl}$, intended to devalue the food pellets, and the other group received the food pellets and $\mathrm{LiCl}$ injections unpaired, which was expected to leave the value of the food pellets intact. Finally, CRs to the light CS were examined, in the absence of food presentations. Unlesioned rats were expected to show lower CR levels after food-LiCL pairings than after unpaired food and LiCL presentations. The question of interest in Experiment $1 \mathrm{~B}$ was whether conditioned responding of ABLlesioned rats would be equally sensitive to the post-training devaluation of the US. If in ABL-lesioned rats, first-order CSs do not gain access to the value of the US, then first-order conditioned responding of those rats would be insensitive to post-training devaluation of the US.

\section{Materials and methods}

Subjects. The 14 lesioned and 19 unlesioned rats from Groups PP and PU of Experiment 1A served as subjects in Experiment 1B. They were maintained at $85 \%$ of their normal weights, as in Experiment $1 \mathrm{~A}$.

Apparatus. The food devaluation phase took place in the rats' stainless steel home cages, and the final test of CRs to the light CS took place in the experimental chambers used in Experiment 1A.

Behavioral testing. After the completion of Experiment 1A, ABLlesioned and unlesioned rats in Groups PP and PU were randomly assigned to two conditions, Devalue (6 ABL-lesioned and 9 unlesioned rats) and Control (8 ABL-lesioned and 10 unlesioned rats). On each of two food aversion training days, all rats first received, in their home cages, $10 \mathrm{~min}$ access to a $10 \mathrm{~cm}$ glass bowl that contained one-hundred $45 \mathrm{mg}$ 


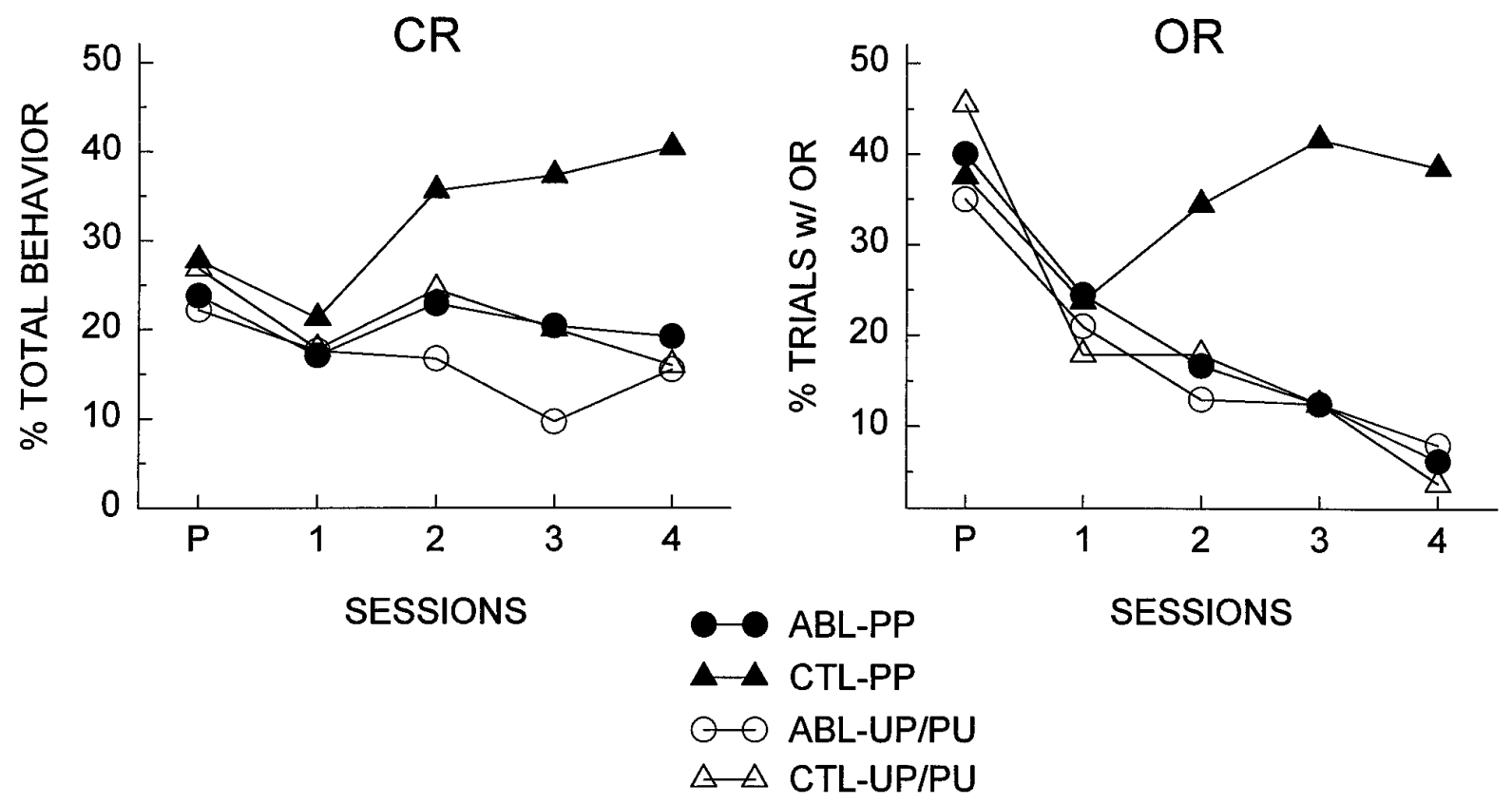

Figure 3. Second-order conditioned responses displayed by rats with basolateral amygdala lesions $(A B L)$ and unlesioned control rats $(C T L)$ during tone presentations in Phase 2 of Experiment 1A. Performance of rats that received both light-food and tone-light pairings (Group $P P)$ is indicated by the solid symbols, and the combined performance of rats that received light-food pairings but no tone-light pairings (Group $P U$ ) and rats that received tone-light pairings but not light-food pairings (Group UP) is indicated by the open symbols. Session $P$ refers to the pretest of the tone at the beginning of Phase 2 .

food pellets (like those used as the US in Experiment 1A). Each rat in the Devalue condition received an injection of $0.3 \mathrm{M} \mathrm{LiCl}$ solution $(5 \mathrm{ml} / \mathrm{kg}$, i.p.) immediately after the food access, whereas rats in the control condition received the same injections $6 \mathrm{hr}$ later. The two aversion sessions were separated by a rest day.

Next, the rats received a single test session in the experimental chambers to examine CRs to the light CS. In this 64 min session, four $10 \mathrm{sec}$ light and four $10 \mathrm{sec}$ tone presentations were randomly intermixed (responding to the tone CSs is not reported here). No food was delivered in this session. The behavioral observation methods used in this test session were the same as those used in Experiment 1A. On the next day, the rats received $10 \mathrm{~min}$ access to one-hundred $45 \mathrm{mg}$ food pellets in the bowl in the home cage (as before), as a final home-cage test of the food aversion. Food consumption (on all of these home-cage trials) was measured by weighing the food pellets before and after each period of food access (including spilled pellets). In addition, a food consumption test was administered in the experimental chamber $2 \mathrm{hr}$ after the completion of the final home cage consumption test. In this test, the rats were given 5 min access to 50 food pellets placed in the experimental chambers' regular food cups. Consumption in this test was determined by counting the number of pellets remaining after the test.

\section{Results and discussion \\ Food aversion}

The ABL lesions did not affect the acquisition of the food aversion. The left side of Figure 4 shows mean food pellet consumption on the two food devaluation trials and the final test trial in the home cages. Food consumption decreased in the Devalued subjects, whether lesioned or unlesioned. Consumption on the final test trial was reliably lower in the Devalued condition than in the Control condition in both ABL-lesioned $\left(U_{(8,6)}=0\right)$ and unlesioned $\left(U_{(10,9)}=0\right)$ rats. Consumption of ABL-lesioned and unlesioned rats did not differ in either Devalued $\left(U_{(9,6)}=24\right)$ or Control $\left(U_{(10,8)}=35\right)$ conditions.

In addition, the food aversion established in the home cages was evident in the experimental chambers as well. In the experimental chamber consumption test, consumption in the Devalued condition averaged 6.0 pellets in the unlesioned rats and 4.0 pellets in the ABL-lesioned rats, whereas consumption in the
Control condition averaged 43.2 pellets in the unlesioned rats and 39.1 pellets in the ABL-lesioned rats. Thus, the ABL lesions did not affect the context generality of the flavor aversion learning. This is an important observation, because it shows that lesioned and unlesioned rats both identified the food pellets received in the experimental chambers with the food pellets received in the home cage bowl in the devaluation phase.

The observation that neurotoxic ABL lesions had no effect on the acquisition of a food aversion is consistent with the results of other research using this lesion method (Dunn and Everitt, 1988; Cahill and McGaugh, 1990; Hatfield et al., 1992). It is worth noting that although several earlier studies that used electrolytic amygdala lesions found disruption of flavor aversion learning (Rolls and Rolls, 1973; Nachman and Ashe, 1974), retrograde transport studies of rats with NMDA or electrolytic lesions indicated that it was the destruction of fibers to/from insular cortex passing through the amygdala, rather than amygdala damage per se that produced flavor aversion deficits with the electrolytic lesions (Dunn and Everitt, 1988).

Recently, Burns et al. (1996) found NMDA lesions of ABL to reduce normal unconditioned neophobic responses to novel food. We did not see any differences in pellet consumption on the first trial of the devaluation phase in Experiment 1B; however, it should be noted that by that time in the experiment the food pellets were not novel.

\section{CRs to light $C S$}

The ABL lesions eliminated the devaluation effect seen with intact rats. The right side of Figure 4 shows food-cup responding to the first-order light CS during the postdevaluation test session. Postconditioning devaluation of the food US reduced CRs to the light CS in unlesioned rats, but not in ABL-lesioned rats. Responding to the light was reliably lower in the Devalued condition than in the Control condition in the unlesioned rats $\left(U_{(10,9)}=\right.$ $14.5)$ but not the ABL-lesioned rats $\left(U_{(8,6)}=27.5\right)$. Furthermore, 


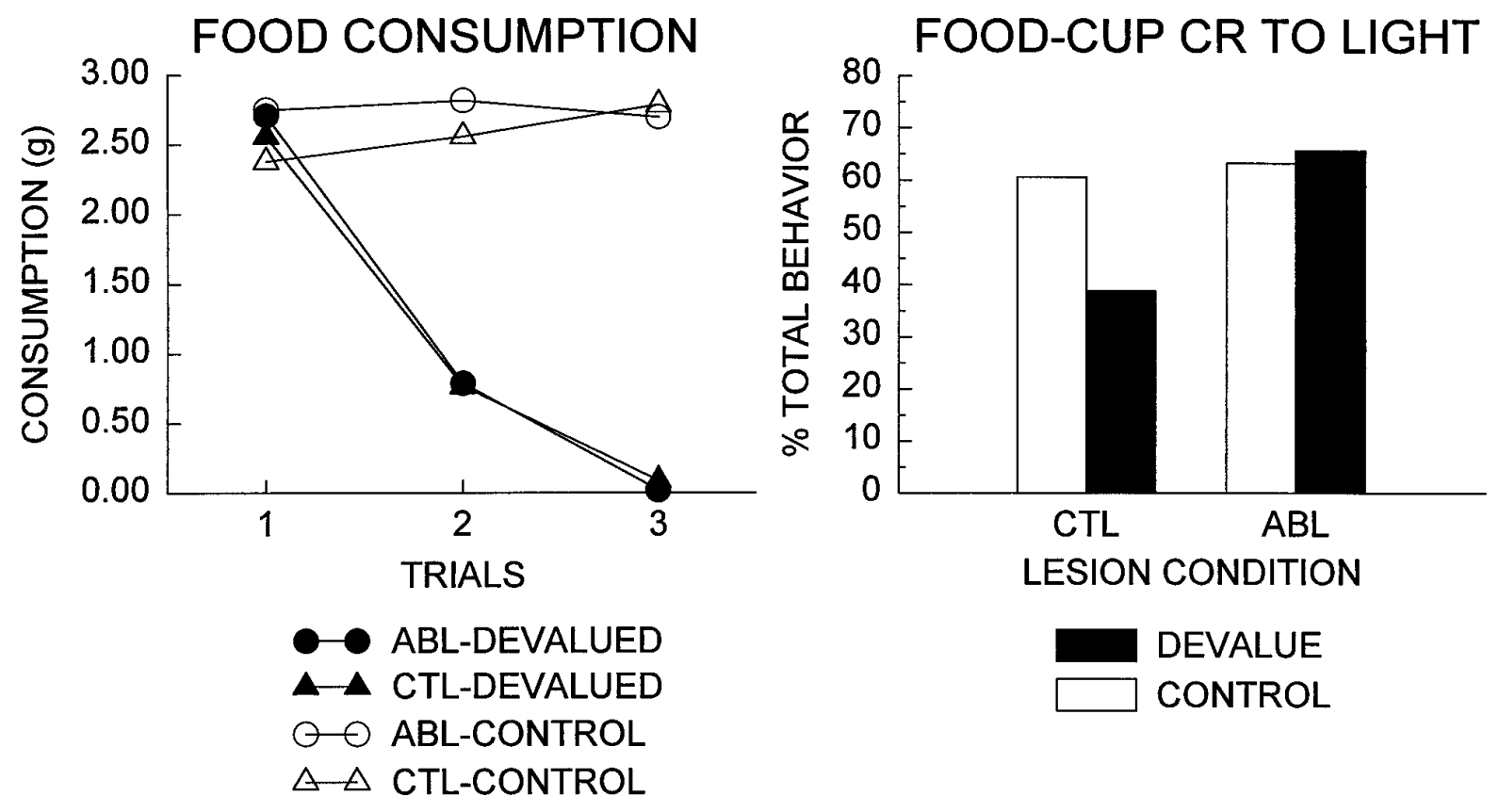

Figure 4. Food consumption in the taste aversion conditioning and test phases of Experiment $1 \mathrm{~B}$ (left) and conditioned food-cup responding to the first-order light CS (right) after taste aversion training. The filled symbols and bars indicate performance of rats for which the food pellets were devalued by pairings with $\mathrm{LiCl}$ injections in the taste aversion conditioning phase, and the open symbols and bars indicate performance of control rats that received unpaired presentation of food and $\mathrm{LiCl}$. $A B L$ refers to rats with basolateral amygdala lesions and $C T L$ to unlesioned rats.

the ABL-lesioned rats in the Devalued condition showed significantly more responding than the unlesioned rats in that condition $\left(U_{(9,6)}=8\right)$.

Thus, although ABL lesions had no effect on the acquisition and display of first-order CRs, the acquisition of a flavor aversion, or the transfer of that aversion to the experimental chamber in which the first-order CRs were learned, they abolished the ability to spontaneously adjust CRs appropriately to post-training alterations in the value of the US.

\section{EXPERIMENT 2A}

Experiment 2A examined the effect of $\mathrm{CN}$ lesions on the acquisition of Pavlovian second-order conditioning, with the same behavioral procedures used in Experiment 1A. If CN lesions, like ABL lesions, interfered with CSs' acquisition of conditioned reinforcement value, then lesioned rats would fail to acquire second-order conditioning.

\section{Materials and methods}

Subjects. Eighty-two experimentally naive male Long-Evans rats (300$350 \mathrm{gm}$ ) that were obtained from Charles River Breeding Laboratories (Raleigh, NC) served as subjects. Rats were individually housed and maintained as described in Experiment 1A.

Apparatus. The apparatus was the same as that used in Experiment 1A. Surgery. Fifty-five rats received CN lesions, and 27 rats served as controls. Bilateral lesions of the $\mathrm{CN}$ were made using the following stereotaxic coordinates: $2.3 \mathrm{~mm}$ posterior from bregma and $4.2 \mathrm{~mm}$ lateral from the midline, with one injection site $7.9 \mathrm{~mm}(0.2 \mu \mathrm{l})$ ventral from the skull surface (Paxinos and Watson, 1986). CN lesions were made with ibotenic acid $(10 \mathrm{mg} / \mathrm{ml})$ in Krebs-Ringer phosphate solution, $\mathrm{pH}$ 7.4. Ibotenic acid was injected using a Hamilton $1.0 \mu$ l syringe at a rate of $0.2 \mu \mathrm{l} / 30 \mathrm{sec}$. Injector needles were left in place at the injection site for 3 min. Control rats received injections of vehicle only. All subjects recovered postoperatively for 2 weeks with access to food and water ad libitum.

Behavioral testing and observations. Behavioral testing and observational procedures were identical to those described in Experiment 1A.

\section{Results and discussion Histology}

A representative photomicrograph of a $\mathrm{CN}$ lesion is shown in Figure 5. Histological analysis of the $\mathrm{CN}$ revealed that 20 of the 55 lesioned rats had neuronal loss that was confined bilaterally to the $\mathrm{CN}$. These lesions ranged in size from 30 to $80 \%$ of the total $\mathrm{CN}$ area, with considerable damage to the medial division of the $\mathrm{CN}$ in all cases. The average lesion encompassed $45 \%$ of the $\mathrm{CN}$. Lesion sites were marked with gliosis, and intact neurons were clearly visible at the borders of the lesions. There was also no detectable loss of neurons in the ABL in the final group of rats with acceptable $\mathrm{CN}$ lesions. No animals were excluded from the control group; injector tracts were visible in all cases. Thus, data for $20 \mathrm{CN}$-lesioned and 27 control animals were analyzed.

\section{Behavior}

Consistent with previous data from our laboratories (Gallagher et al., 1990), first-order food-cup CRs were acquired rapidly to the light CS when it was paired with food (Groups PP and PU) regardless of lesion condition, but conditioned ORs (rear behavior) were acquired only in the intact rats. Figure 6 shows performance of the first-order CRs and conditioned ORs during the light CS on light-food or light-alone reminder trials in Phase 2.

The left side of Figure 6 shows food-cup CRs. Both $\mathrm{CN}$ lesioned $\left(U_{(17,3)}=0\right)$ and unlesioned $\left(U_{(23,4)}=0\right)$ rats in Groups PP and PU (combined) showed reliably more food-cup responding than those in Group UP, in which the light and food were explicitly unpaired. Performance of lesioned and intact rats did not differ in either the paired $\left(U_{(17,23)}=182\right)$ or the unpaired $\left(U_{(3,4)}=5\right)$ rats.

The right side of Figure 6 shows conditioned ORs (rear behavior). The unlesioned rats in Groups PP and PU (combined) showed reliably more rear behavior than those in Group UP 

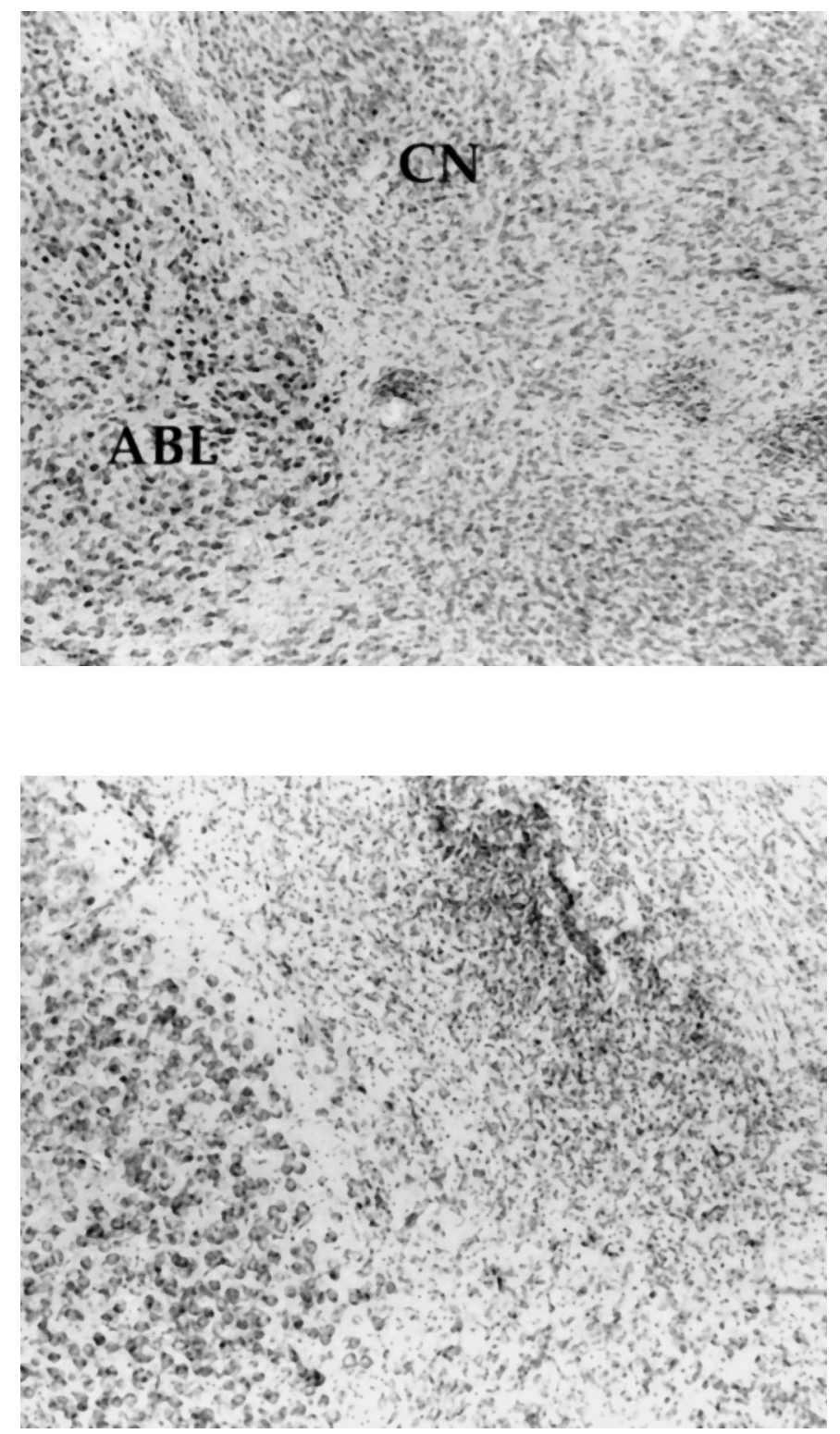

Figure 5. Photomicrographs showing the region of basolateral amygdala $(A B L)$ and amygdala central nucleus $(C N)$ in a vehicle-injected control brain (top panel) and in a $\mathrm{CN}$ ibotenate-lesioned brain (bottom panel). Note neuron loss and gliosis at the $C N$ lesion site, and sparing of neurons in $A B L$.

$\left(U_{(23,4)}=11\right)$. In contrast, the CN-lesioned rats in Groups PP and PU did not show reliably higher levels of rear behavior than those in Group UP $\left(U_{(17,3)}=22\right)$. Rear behavior was significantly more frequent in Groups PP and PU among unlesioned rats than among lesioned rats $\left(U_{(17,23)}=92\right)$.

Figure 7 shows the primary behavioral data of Experiment 2A, the acquisition of second-order conditioning to the tone CS in Phase 2. The left side of Figure 7 shows the acquisition of second-order CRs (food-cup and walk behaviors combined). In Group PP these second-order CRs were acquired in both $\mathrm{CN}$ lesioned and unlesioned rats; responding of lesioned and unlesioned rats in Group PP did not differ significantly over the course of Phase $2\left(U_{(10,14)}=59\right)$. As expected, rats in the two behavioral control groups (PU and UP, combined) did not acquire these second-order CRs. Responding of Group PP was reliably greater

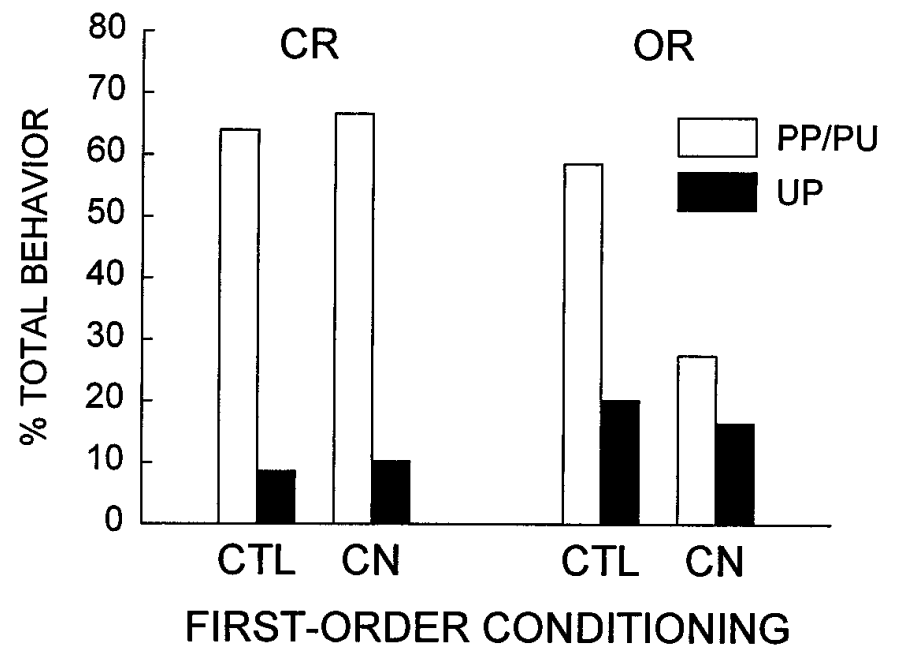

Figure 6. First-order conditioned responses displayed by rats with central nucleus lesions $(C N)$ and unlesioned control rats $(C T L)$ during the light reminder trials in Phase 2 of Experiment 2A. Combined performance of the rats that received light-food pairings (Groups $P P$ and $P U$ ) is indicated by the open bars and performance of the rats that received unpaired presentations of light and food (Group UP) is indicated by the solid bars.

than that of the combined controls in both unlesioned $\left(U_{(14,13)}=\right.$ $3)$ and lesioned $\left(U_{(10,10)}=19\right)$ rats. Thus, $\mathrm{CN}$ damage did not significantly affect the acquisition of second-order CRs.

The right side of Figure 7 shows the acquisition of second-order conditioned ORs (startle) to the tone CS. The unlesioned rats in Group PP acquired conditioned ORs, displaying reliably more ORs over the course of tone-light pairings than the unlesioned rats in the combined control groups $\left(U_{(14,13)}=21.5\right)$. In contrast, there was no evidence for the acquisition of conditioned ORs in the CN-lesioned rats of Group PP: those rats did not show reliably more ORs than the $\mathrm{CN}$-lesioned rats in the combined control groups $\left(U_{(10,10)}=42\right)$. Unlesioned rats in Group PP showed significantly more OR behavior than lesioned rats in that group $\left(U_{(10,14)}=33\right)$. Thus, $\mathrm{CN}$ damage interfered with the acquisition of second-order conditioned ORs, just as it interfered with firstorder conditioned ORs.

The normal acquisition of first-order CRs to the light CS and second-order CRs to the tone CS observed here in rats with $\mathrm{CN}$ lesions indicates that the amygdala $\mathrm{CN}$ is not importantly involved in the acquisition of reinforcing power by Pavlovian CSs, despite its role in the acquisition of both first- and second-order conditioned ORs. These data complement those of Robledo et al. (1994), who reported that rats with ibotenic lesions of amygdala $\mathrm{CN}$ were not impaired in the acquisition of an operant response in a secondary reinforcement procedure.

\section{EXPERIMENT 2B}

In Experiment 2A, CN-lesioned rats showed normal acquisition of second-order CRs, suggesting that $\mathrm{CN}$ lesions did not interfere with the acquisition of reinforcement value by the first-order light CS paired with food. Experiment 2B considered whether $\mathrm{CN}$ lesions interfered with CSs' access to the current value of the US by using a devaluation procedure identical to that used in Experiment 1B. If in CN-lesioned rats, as in normal rats, first-order CSs have access to the current value of the US, then first-order conditioned responding of $\mathrm{CN}$-lesioned and normal rats might be equally sensitive to post-training devaluation of the US. 


\section{CR}

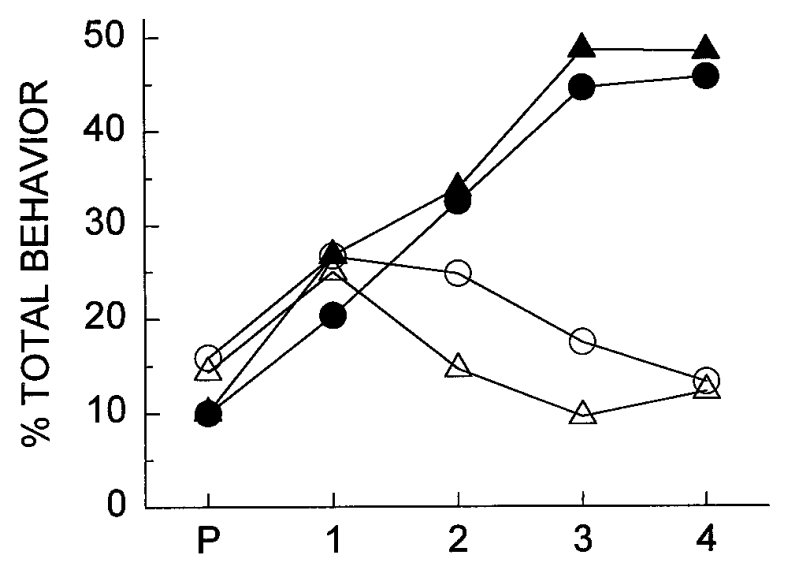

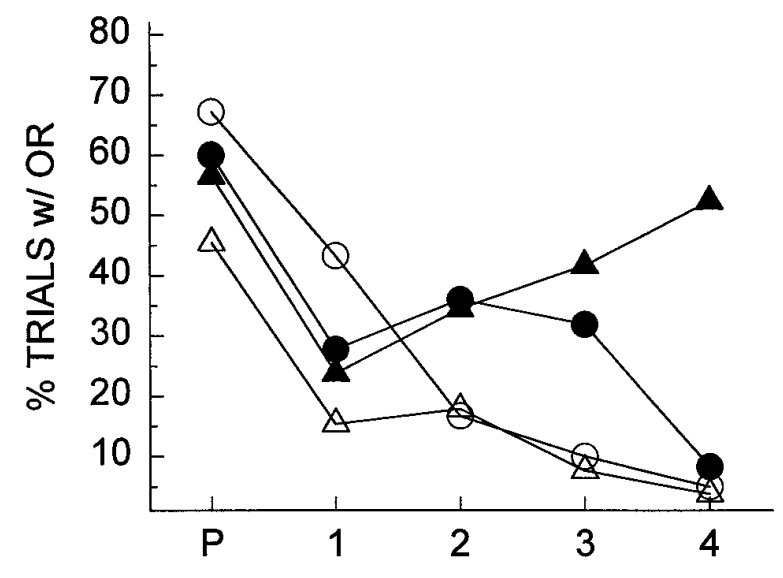

SESSIONS

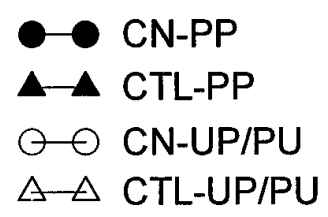

Figure 7. Second-order conditioned responses displayed by rats with central nucleus lesions $(C N)$ and unlesioned control rats $(C T L)$ during tone presentations in Phase 2 of Experiment 2A. Performance of rats that received both light-food and tone-light pairings (Group PP) is indicated by the solid symbols, and the combined performance of rats that received light-food pairings but no tone-light pairings (Group $P U$ ) and rats that received tone-light pairings but not light-food pairings (Group UP) is indicated by the open symbols. Session $P$ refers to the pretest of the tone at the beginning of Phase 2.

\section{Materials and methods}

Subjects. All rats from Groups PP and PU of Experiment 2A, $17 \mathrm{CN}-$ lesioned and 23 unlesioned, served as subjects.

Apparatus. The apparatus was the same as that described in Experiment $1 \mathrm{~A}$, except that 6 -cm-diameter crucibles replaced the glass bowls.

Behavioral testing and observations. The behavioral testing and observations procedures were the same as those described in Experiment 1B, except for the deletion of the final food consumption test in the experimental chamber.

\section{Results}

\section{Food aversion}

Consistent with previous findings of Bermudez-Rattoni et al. (1986) and Hatfield et al. (1992), the CN lesions had no effect on the acquisition of the food aversion. The left panel of Figure 8 shows mean food pellet consumption on the two food devaluation trials and the final test trial in the home cages. Food consumption decreased dramatically in the Devalued subjects, whether lesioned or unlesioned. Consumption on the final test trial was reliably lower in the Devalued condition than in the Control condition in both $\mathrm{CN}$-lesioned $\left(U_{(8,9)}=0\right)$ and unlesioned $\left(U_{(12,11)}=0\right)$ rats. Consumption of $\mathrm{CN}$-lesioned and unlesioned rats did not differ in either the Devalued $\left(U_{(8,12)}=47\right)$ or Control $\left(U_{(9,11)}=47\right)$ conditions.

\section{CRs to light $C S$}

The $\mathrm{CN}$ lesion did not alter the devaluation effect. The right side of Figure 8 shows food-cup responding to the first-order light CS during the postdevaluation test session. Postconditioning devaluation of the food US reduced CRs to the light CS in both lesioned and unlesioned rats. Responding to the light was reliably lower in the Devalued condition than in the Control condition in both lesioned $\left(U_{(8,9)}=15\right)$ and unlesioned $\left(U_{(12,11)}=30\right)$ rats. Performance of lesioned and unlesioned rats did not differ significantly in either the Devalued $\left(U_{(8,12)}=38\right)$ or the Control $\left(U_{(9,11)}=\right.$
45.5) condition. Thus, the ability to spontaneously adjust CRs appropriately to alterations in the value of the US was not affected by $\mathrm{CN}$ lesions.

The results of Experiments $2 \mathrm{~A}$ and $2 \mathrm{~B}$ demonstrate that $\mathrm{CN}$ lesions have no discernible effect on the ability of cues to acquire reinforcement value or gain access to the current value of the US in the production of learned responses. The same lesions, however, impaired conditioned orienting, one indicator of the role of this structure in the regulation of attentional processing of cues in associative learning. The results of an earlier study (Gallagher and Holland, 1992) further support these claims. In that experiment, we tested rats with neurotoxic $\mathrm{CN}$ lesions in another procedure used to examine the motivational value acquired by cues in Pavlovian conditioning (Weingarten, 1983). First, a light CS was paired with food while the rats were food-deprived. In that phase, the normal but not the $\mathrm{CN}$-lesioned rats displayed conditioned ORs. Next, the rats were fully satiated and then tested for food consumption in the presence or absence of the light CS. Light presentations produced a robust increase in food consumption in both normal and $\mathrm{CN}$-lesioned rats. All of these findings are consistent with the claim that the amygdala $\mathrm{CN}$ is involved in the acquisition of conditioned ORs, but not the transfer of value from the US to the CS in Pavlovian appetitive conditioning.

\section{GENERAL DISCUSSION}

Lesions of basolateral amygdala interfered with both the acquisition of Pavlovian appetitive second-order conditioning (Experiment 1A) and the sensitivity of first-order CRs to postconditioning devaluation of the food US (Experiment 1B). At the same time, there was no evidence that ABL lesions interfered with the acquisition of either light-food or food-toxin associations or the acquisition of conditioned ORs to light CSs paired with food. Thus, these results join the growing body of evidence that impli- 


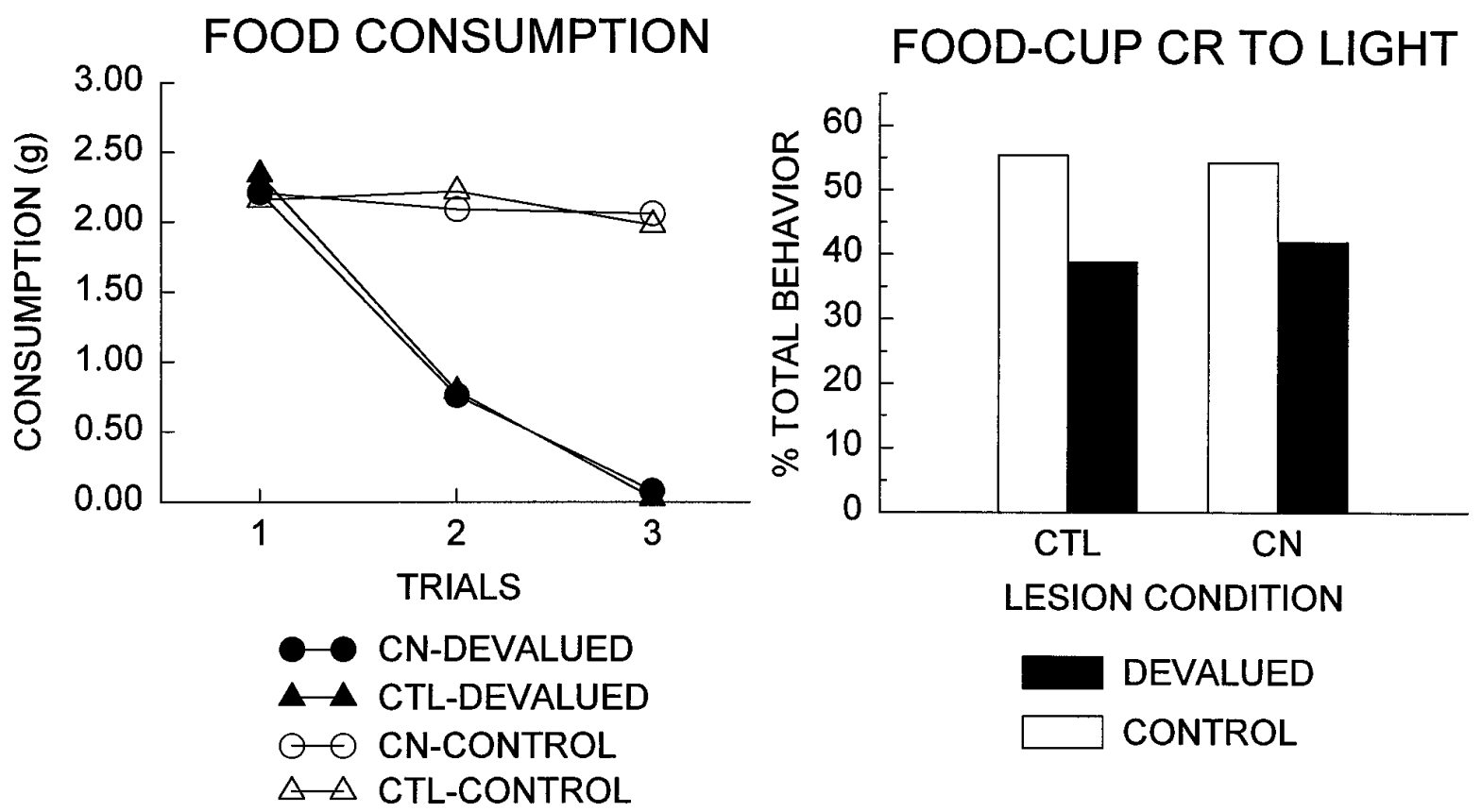

Figure 8. Food consumption in the taste aversion conditioning and test phases of Experiment 2B (left) and conditioned food-cup responding to the first-order light CS (right) after taste aversion training. The filled symbols and bars indicate performance of rats for which the food pellets were devalued by pairings with $\mathrm{LiCl}$ injections in the taste aversion conditioning phase, and the open symbols and bars indicate performance of control rats that received unpaired presentation of food and $\mathrm{LiCl}$. $\mathrm{CN}$ refers to rats with central nucleus lesions and CTL to unlesioned rats.

cates $\mathrm{ABL}$ in associative learning processes that give CSs access to the motivational value of their associated USs (Everitt and Robbins, 1992).

The results of Experiment 2A replicated the findings of Gallagher et al. (1990): lesions of amygdala CN impaired the acquisition of Pavlovian conditioned ORs to a light paired with food, but had no effect on the acquisition of conditioned behavior directed toward the food cup. They extend the findings of Gallagher et al. (1990) by showing that CN lesions also impair the acquisition of second-order conditioned ORs, but do not affect the acquisition of second-order food-cup CRs. In addition, in Experiment 2B, $\mathrm{CN}$ lesions had no effect on the sensitivity of first-order food-cup responses to postconditioning changes in the value of the US. Thus, it seems reasonable to assert that $\mathrm{CN}$ is not importantly involved in the assignment of value or significance to CSs in this Pavlovian appetitive conditioning preparation.

In contrast to our findings with appetitive conditioning procedures, in aversive conditioning procedures the $\mathrm{CN}$ appears to be critical for the expression of conditioned behaviors usually described as emotional responses. Freezing behavior, potentiation of the startle reflex, and many autonomic indices of fear depend on intact projections from amygdala $\mathrm{CN}$ to brainstem systems that provide output for these responses. Thus, although we found little evidence for $\mathrm{CN}$ involvement in the CSs' acquisition of positive incentive value, $\mathrm{CN}$ is clearly involved in other aspects of learned emotional function.

Nevertheless, the present findings support the view that separate amygdala subsystems mediate attentional processes and the acquisition of cue value during conditioning (Gallagher and Holland, 1992, 1994). Although this proposal was based on the results of a variety of lesion studies, it is worth noting that electrophysiological data from rats in appetitive conditioning experiments led Muramoto et al. (1993) to a similar suggestion. They found that single neurons in basolateral amygdala were more likely to ac- quire patterns of activity to CSs that were similar to those generated initially by the US than were neurons in corticomedial amygdala (including $\mathrm{CN}$ ). Those same neurons were also more likely to show sensitivity to the affective nature of the USs, displaying opposite patterns of activity to appetitive and aversive USs, and to their corresponding CSs.

Additional lines of research are beginning to define the importance of connections of $\mathrm{ABL}$ and $\mathrm{CN}$ with other brain systems in the mediation of changes in attention and cue value. For example, the basolateral region of the amygdala is connected with ventral striatum, including a direct innervation of nucleus accumbens. This projection appears to play an important role in the associative process by which cues acquire reinforcement value. Everitt et al. (1991) examined rats' acquisition of a preference for a distinctive location paired with delivery of a sucrose reinforcer after various lesions of amygdala or striatum. The place preference exhibited by normal rats was reduced after bilateral quinolinate lesions of basolateral amygdala, after bilateral quisqualate lesions of ventral striatum, and after asymmetrical lesions of the basolateral amygdala and ventral striatum, which functionally disconnected the two regions despite producing only unilateral damage to each structure. The detrimental effect of the asymmetrical lesion especially supports the view that connections between the basolateral amygdala and ventral striatum are critical for processes whereby cues acquire reinforcing value. In addition, this role for connectivity between the basolateral amygdala and ventral striatum would allow for changes in the emotional significance of CSs in associative learning to proceed independently of the amygdala $\mathrm{CN}$.

Research from our laboratories has implicated other projections originating in the central nucleus with the regulation of conditioned orienting and changes in CS associability. Han et al. (1995) found evidence that conditioned orienting depends on an amygdalo-nigrastriatal circuit, which originates in the amygdala 
$\mathrm{CN}$ and includes dopaminergic innervation of the dorsolateral striatum. Rats with unilateral ibotenate lesions of amygdala $\mathrm{CN}$ and 6-OHDA lesions of the contralateral striatum, like bilaterally lesioned $\mathrm{CN}$ rats, were impaired in the acquisition of conditioned ORs but not conditioned food-cup behavior. Unilateral lesions of either $\mathrm{CN}$ alone or striatum alone had no effects.

Additional evidence indicates that amygdala $\mathrm{CN}$ regulates attentional processing of cues during associative learning through its projection to magnocellular cholinergic neurons in the basal forebrain, which in turn project to cortex. Chiba et al. (1995) found that immunotoxic lesions of those cholinergic neurons reproduced an effect of $\mathrm{CN}$ damage on attentional processing observed earlier (Holland and Gallagher, 1993a). Shifts in the predictive accuracy of a CS disrupted subsequent conditioning with that cue after both $\mathrm{CN}$ and cholinergic lesions, whereas those shifts enhanced conditioning in intact rats. Rats with cholinergic lesions, in contrast to those with $\mathrm{CN}$ lesions, showed no deficit in conditioned orienting, a dissociation consistent with our observation that those behavioral orienting responses depend on another output from the amygdala $\mathrm{CN}$ that regulates dorsolateral striatal function.

The studies just described suggest that $\mathrm{CN}$ connectivity with the forebrain plays a role in the regulation of attention and aspects of information processing. The present findings are consistent with the view that those pathways and functions of the amygdala are separable from ones that provide a substrate for the positive value that cues acquire in Pavlovian appetitive conditioning. Thus, in addition to their well recognized role in emotional learning, the different subsystems within the amygdala may provide linkages between affective and cognitive processing. Better understanding of these linkages may shed light on the relations between various cognitive and affective disorders.

\section{REFERENCES}

Bermudez-Rattoni F, Grijalva CV, Kiefer SW, Garcia J (1986) Flavorillness aversions: the role of the amygdala in the acquisition of tastepotentiated odor aversions. Physiol Behav 38:503-508.

Burns LH, Annett L, Kelley AE, Everitt BJ, Robbins TW (1996) Effects of lesions to amygdala, ventral subiculum, medial prefrontal cortex and nucleus accumbens on the reaction to novelty: implications for limbicstriatal interactions. Behav Neurosci 110:60-73.

Cahill L, McGaugh JL (1990) Amygdaloid complex lesions differentially affect retention of tasks using appetitive and aversive reinforcement. Behav Neurosci 104:532-543.

Chiba AA, Bucci DJ, Holland PC, Gallagher M (1995) Basal forebrain cholinergic lesions disrupt increments but not decrements in conditioned stimulus processing. J Neurosci 15:7315-7322.

Davis M (1992) The role of the amygdala in conditioned fear. In: The amygdala: neurological aspects of emotion, memory, and mental dysfunction (Aggleton J, ed), pp 255-306. Chichester: Wiley.

Dunn LT, Everitt BJ (1988) Double dissociations of the effects of amygdala and insular cortex lesions on conditioned taste aversion, passive avoidance and neophobia in the rat using the excitotoxin ibotenic acid. Behav Neurosci 102:3-23.

Everitt BJ, Robbins TW (1992) Amygdala-ventral striatal interactions and reward-related processes. In: The amygdala: neurobiological aspects of emotion, memory, and mental dysfunction (Aggleton J, ed), pp 401-429. New York: Wiley.
Everitt BJ, Morris KA, O'Brien A, Robbins TW (1991) The basolateral amygdala-ventral striatal system and conditioned place preference: further evidence of limbic-striatal interactions underlying reward-related processes. Neuroscience 42:1-18.

Gallagher M, Holland PC (1992) Understanding the function of the central nucleus: is simple conditioning enough? In: The amygdala (Aggleton J, ed), pp 307-321. New York: Wiley.

Gallagher M, Holland PC (1994) The amygdala complex: multiple roles in associative learning and attention. Proc Natl Acad Sci USA 91:11771-11776.

Gallagher M, Graham PW, Holland PC (1990) The amygdala central nucleus and appetitive Pavlovian conditioning: lesions impair one class of conditioned behavior. J Neurosci 10:1906-1911.

Han JS, McMahan RW, Holland PC, Gallagher M (1995) Conditioned orienting behavior is mediated by the amygdalo-nigrostriatal pathway. Soc Neurosci Abstr 21:1224.

Hatfield T, Graham PW, Gallagher M (1992) Taste-potentiated odor aversion learning: role of the amygdaloid basolateral complex and central nucleus. Behav Neurosci 106:286-293.

Holland PC (1977) Conditioned stimulus as a determinant of the form of the Pavlovian conditioned response. J Exp Psychol 3:77-104.

Holland PC (1993) Cognitive aspects of Pavlovian conditioning. Curr Opin Neurobiol 3:230-236.

Holland PC, Gallagher M (1993a) Amygdala central nucleus lesions disrupt increments, but not decrements, in conditioned stimulus processing. Behav Neurosci 107:246-253.

Holland PC, Gallagher M (1993b) The effects of amygdala central nucleus lesions on blocking and unblocking. Behav Neurosci 107:235-245.

Holland PC, Rescorla RA (1975) Second-order conditioning with food unconditioned stimulus. J Comp Physiol Psychol 88:459-467.

Holland PC, Straub JJ (1979) Differential effects of two ways of devaluing the unconditioned stimulus after Pavlovian appetitive conditioning. J Exp Psychol 5:65-78.

Killcross AS, Everitt BJ, Robbins TW (1995) Dissociable effects of selective excitotoxic lesions of the amygdala on instrumental and Pavlovian components of a novel conditioned punishment procedure. Soc Neurosci Abstr 21:1667.

LeDoux JE (1992) Emotion and the amygdala. In: The amygdala: neurobiological aspects of emotion, memory, and mental dysfunction (Aggleton J, ed). New York: Wiley.

Mackintosh NJ (1983) Conditioning and associative learning. Oxford: Clarendon.

McDonald RJ, White NM (1993) A triple dissociation of memory systems: hippocampus, amygdala, and dorsal striatum. Behav Neurosci 107:3-22.

Muramoto K, Ono T, Nishijo H, Fukuda M (1993) Rat amygdaloid neuron responses during auditory discrimination. Neuroscience 52:621-636.

Nachman M, Ashe JH (1974) Effects of basolateral amygdala lesions on neophobia, learned taste aversions and sodium appetite in rats. J Comp Physiol Psychol 87:622-643.

Paxinos G, Watson C (1986) The rat brain in stereotaxic coordinates. New York: Academic.

Robledo P, Robbins TW, Everitt BJ (1994) Effects of ibotenic acid lesions of the central nucleus of the amygdala on potentiated responding with conditioned reinforcement following intra-accumbens amphetamine. Behav Pharmacol 5:105.

Rolls ET, Rolls BJ (1973) Altered food preferences after lesions in the basolateral region of the amygdala in the rat. J Comp Physiol Psychol 83:248-259.

Weingarten HP (1983) Conditioned cues elicit feeding in sated rats: a role for learning in meal initiation. Science 220:431-433. 